\title{
NUMERICAL MODELING OF FRETTING WEAR
}

\author{
T. Yue, M. Abdel Wahab, R. Hojjati-Talemi and M.Z. Sadeghi \\ Ghent University, Laboratory Soete, Belgium
}

\begin{abstract}
In this paper, a review of some techniques proposed in the literature for modelling fretting wear is presented. Fretting wear occurs when surfaces are degraded and materials are removed due to a small relative oscillatory motion between two contact surfaces. Due to the continuous change in the shape of a structure during its lifetime, the design of engineering components subjected to fretting wear is a challenge to engineers and scientists. The available predictive tools in the literature, which make use of analytical solution of the contact pressure in the first cycle ignoring the wear effect and removal of materials in the subsequence cycles, provide inaccurate results. Numerical modelling techniques such as Finite Element Analysis (FEA) can be used to model any type of structures with configuration taking into account many complicated details such as large deformation, material non-linearity, change in geometry and time integration effect. Therefore, FEA is a very desirable technique for fretting wear analysis and lifetime prediction. This review covers modelling fretting wear using numerical modelling methods, which can be divided into three main techniques, namely the FEA method, Boundary Element Method (BEM) and Finite Discrete Element Method (FDEM).
\end{abstract}

Keywords: Fretting wear, Finite Element Method, Contact mechanics

\section{INTRODUCTION}

Fretting wear is a phenomenon, in which surface degradations and removal of materials take place due to a small relative oscillatory motion and quasi-static load between two contact surfaces. There are many parameters that affect fretting wear including normal load, contact stress, frequency, slip amplitude, material properties, surface roughness of the contact bodies, etc. The design of engineering components subjected to fretting wear, such as couplings and splines, jointed structures etc. [1], is still a challenge to engineers. This is because of the continuous change in the component shape during fretting wear. Therefore, a predictive technique that takes into account the wear progress during life cycle is desirable. Analytical solutions of wear problems are very difficult and limited to simple 2-D configuration steady state analysis [2]. In contrast, numerical modeling techniques such as finite element analysis (FEA), boundary element analysis (BEA) can be used for any type of structures in 3-D configuration taking into account many complicated details such as large deformation, material non-linearity, change in geometry and time integration effect. In this paper different numerical modeling methods of fretting wear are reviewed.

\section{FEA MODELING}

With the rapid development of high performance computers and the methods for solving non-linear problems, FEA have applied to simulate the process of fretting wear. Simulation of wear involves solution of a general contact problem, which is highly non-linear due to the contact boundary conditions between the interfaces [3].

In the last ten years literature, there are two main methods to simulate the process of fretting wear according to the wear model used. One is based on Archard's equation to predict evolution of contact geometry and contact variables in the interface with or without the consideration of effects of debris. However, there is underestimation of wear volume compared with experimental values [4,5]. Introducing an energy wear concept to predict wear kinetics and geometrical changes of the wear scar is another kind of method, while the maximum wear depth is underestimated because of ignoring debris [6]. Stéphanie Basseville et al [7] proposed a model to introduce improved models of the contact surface in fretting conditions with energy wear concept, which takes into account the debris but without the cyclic material response, cyclic plasticity and oxidation.

The flowchart of most fretting wear simulation tools is shown as Figue.1. Once initial parameters such as material properties, normal force, sliding amplitude, coefficient of friction, total wear cycles $N$ etc. has been input, the unworn geometry FE model is generated. Then the contact variables such as contact pressure and slip displacement can be calculated by FE software. At each node in the contact surface, the incremetal wear depth $\Delta h$ can be calculated by Archard model or engergy concept. After updating the nodal coordinates and remeshing, the corresponding worn surface profiles and the evolution of surface variables can be predicted. 


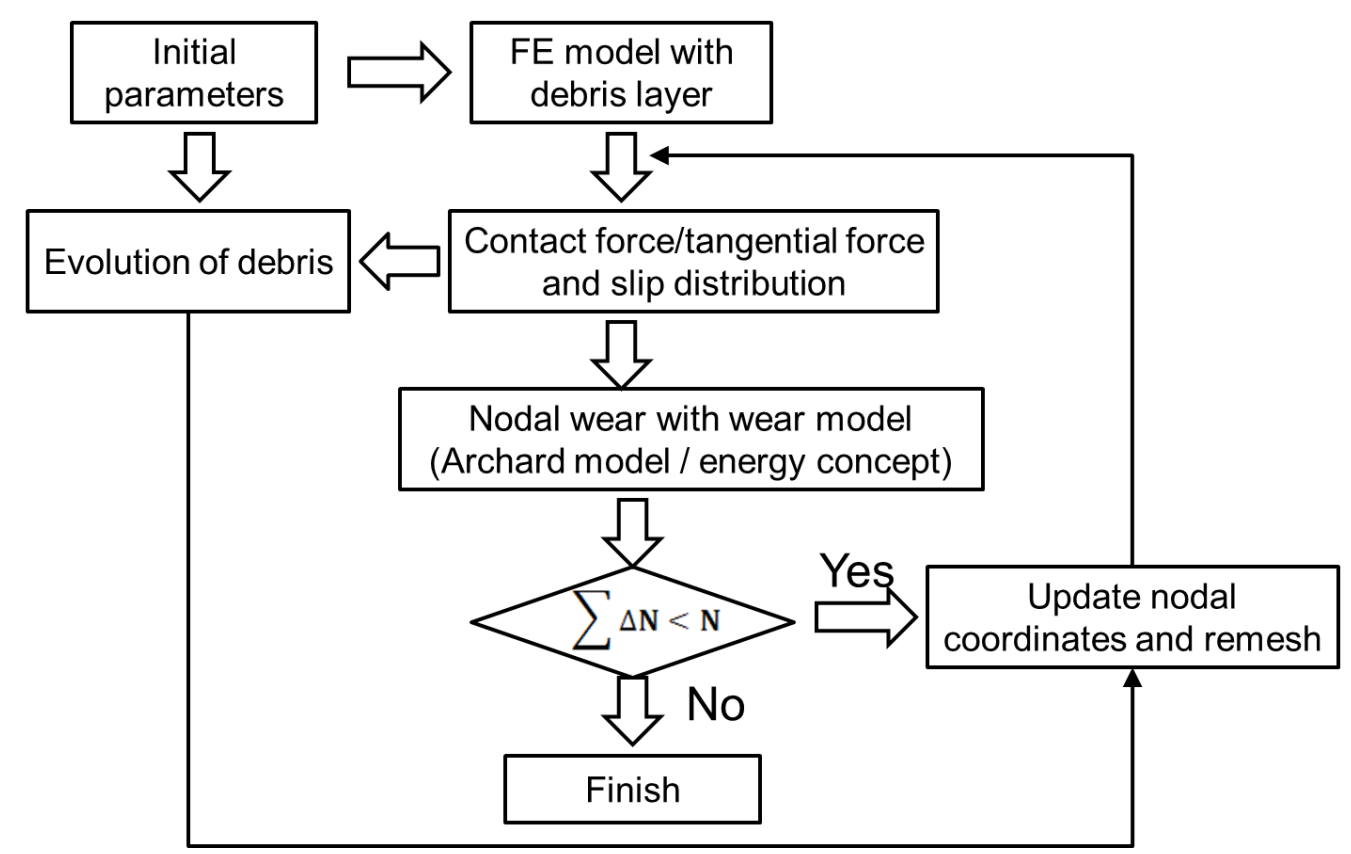

Figue 1. Flowchart of fretting wear simulation tools[5]

\subsection{Archard Model}

The linear wear model that was initially proposed by Archard [8], has been widely used in the literature due to its simplicity. It is usually expressed as:

$$
\frac{V}{S}=K \frac{F}{H}
$$

Where $V$ is the wear volume, $S$ is the sliding distance, $K$ is the Archard wear coefficient, $F$ is the normal force and $H$ is the hardness of the material.

Reference [1] firstly described details of the numerical approach of fretting wear based on Archard model, which predicts the frictional contact behaviour of a cylinder-on-flat fretting test configuration. At node $i$ on the contact surface for the $j$ th wear increment, the contact pressure $P_{i, j}$ and slip per cycle are calculated by FE model. The increment of wear depth for the flat at node $i$ in $j$ th increment $\Delta h_{i, j}$ is given by:

$$
\Delta h_{i, j}=k_{i} \times 4 s_{i, j} \times \Delta N \times P_{i, j}
$$

Where $k_{i}$ is the local Archard wear coefficient, $s_{i, j}$ is the contact displacement at $1 / 4$ cycle at node $i$ in $j$ th increment, $\Delta N$ is the increment in number of simulation wear cycles, and $P_{i, j}$ is the contact pressure at node $i$ in $j$ th increment.

The updated vertical coordinate of flat of node $i$ at $j+1$ th increment $y_{i, j+1}$ is given by:

$$
y_{i, j+1}=y_{i, j}-\Delta h_{i, j}
$$

Where $y_{i, j+1}$ is the vertical coordinate of flat at node $i$ at $j$ th increment.

Repetition of these calculations of the total number of wear cycles achieves the wear simulations.

Based on the wear simulation tool above, the evolution of sub-surface stress fields subjected to fretting wear is investigated under both partial slip and gross slip regimes [4].

A FE method based on Archard model is developed to predict the effect of fretting wear on fretting fatigue life in the rounded edge punch-on-flat geometry in 2007 [9]. The fretting fatigue damage during material removal is predicted by combination of Smith-Watson-Topper parameter with a damage approach, comparing predictions with experimental data.

An elastic-plastic FE method is employed to investigate the evolution of the fretting variables in surface and subsurface [10]. The special attention was given to the evolution of plastic variables and effects of plasticity during fretting wear, using a kinematic hardening plasticity model to present the cyclic plasticity behaviour. 


\subsection{Dissipated Energy Method}

The relationship between fretting wear and dissipated energy is defined as [11,12]:

$$
E d_{c_{(i)}}^{(\lambda)}=E d_{(i)} G\left(\frac{\delta_{g_{(i)}}}{\delta_{g_{r e f}}}, \lambda\right)
$$

Where $E d_{c_{(i)}}^{(\lambda)}, E d_{(i)}$ are the composite energy dissipated and the dissipated energy at the th cycle respectively. $G$ is the function expressing the relative impact of the sliding amplitude on the energy formulation, in which the non-dimensional variable $\lambda$ indexs the adhesive wear property of the contact, $\delta_{g_{(i)}}$ is the applied sliding amplitude, and $\delta_{g_{r e f}}$ is the reference sliding amplitude chosen to normalize the relative impact of sliding amplitude.

If $\lambda=1$, it is a full adhesive wear contact, and $G$ is expressed as:

$$
G\left(\frac{\delta_{g_{(i)}}}{\delta_{g_{r e f}}}, \lambda\right)=\frac{\delta_{g_{(i)}}}{\delta_{g_{r e f}}}
$$

Then

$$
E d_{c}^{(1)}=E d_{s}=\frac{\delta_{g_{(i)}}}{\delta_{g_{\text {ref }}}} E d
$$

Where $E d_{s}$ is the sliding reduced energy, and $E d$ is the dissipated energy. For materals which are sensitve to adhesive phenomena, such as titanium and aluminen alloy, the debris generated at the interfaces can not be elimited easily. Due to adhesive mechanisms, a significant part of dissipated energy is consumed to move and eject debris of interfaces ( Figue 2 ). The higher the slip amplitude applied, the debris can eject more easily. Therefore dissipated energy method can highlight the effect of debris on fretting wear for adhesive materials, which is made possible to simulate fretting wear with the presence of debris.

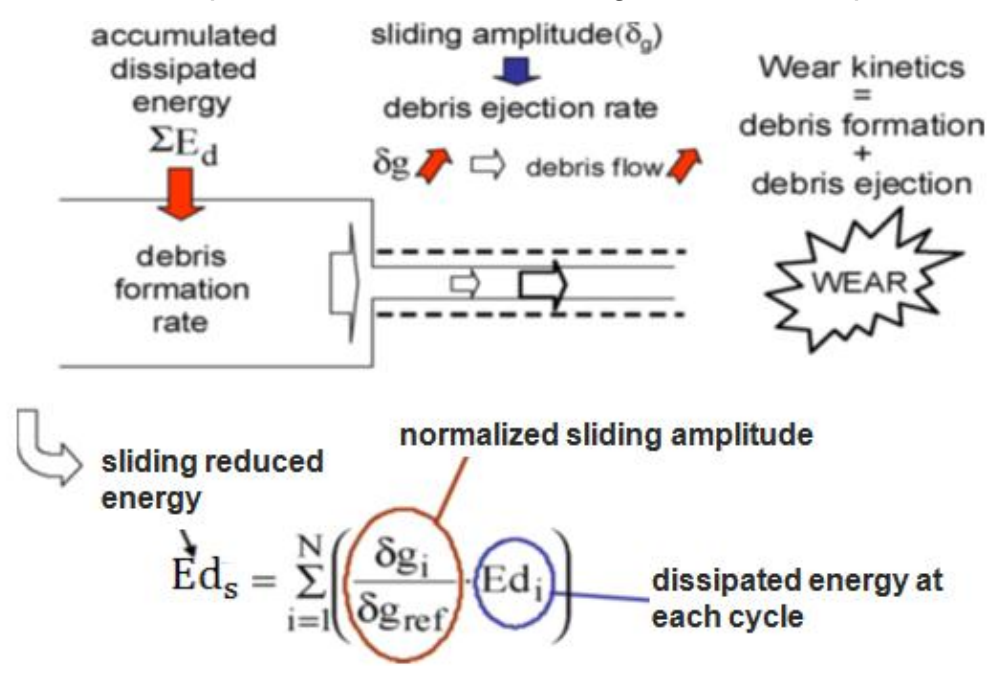

Figue 2. Illustration showing the relations of fretting wear and dissipated energy $[12,13]$

If $\lambda=0$, it is a non-adhesive wear contact, in which the debris can eject immediately. $G$ is expressed as:

$$
\begin{gathered}
G\left(\frac{\delta_{g_{(i)}}}{\delta_{g_{r e f}}}, \lambda\right)=1 \\
E d_{c}^{(0)}=E d
\end{gathered}
$$

This formulation (eq. (4)) is a general formulation to express the wear process whatever the adhesive wear properties of the interface and it also confirms the physical relationships linking the dissipated energy to quantity of damaged material.

Reference [13] presented an FE technique to predict fretting wear of Ti-6Al-4V based on dissipated energy method. 
The reference contact condition (a single sliding amplitude and normal load) is considered to simplify the analysis, therefore the total wear volume $V$ is expressed as:

$$
V=\alpha \sum_{i=1}^{N} E d_{i}
$$

Where $\alpha$ is the energy wear volume coefficient of the studied interface for a given sliding amplitude.

Considering an incremental formulation, increment of wear depth for the flat at $x$ position in ith fretting cycle $\Delta h_{i}(x)$ is given by:

$$
\Delta h_{i}(x)=\alpha_{h} E d_{i}(x)=\alpha_{h} \int_{t=0}^{T} q_{i}(x) d s_{i}(x)
$$

Where $\alpha_{h}$ is local energy wear coefficient, $E d_{i}(x), q_{i}(x), s_{i}(x)$ are the dissipated energy, tangential force and relative sliding at $x$ position at ith fretting cycle of period $T$ respectively.

The updated vertical coordinate of flat of at $x$ position at $i+1$ th fretting cycle is given by:

$$
\Delta y_{i+1}(x)=y_{i}(x)-\Delta h_{i}(x)
$$

And the accumulated dissipated energy is updated by the relation as below:

$$
\sum E d_{i}(x)=\sum E d_{i-1}(x)+E d_{i}(x)
$$

Based on the formulations above the wear profile is predicted.

Reference [14] presented a FE method based on energy method to compare differences between different contact geometries under the same normal load, stroke, etc. This method is able to predict evolution of contact geometry, wear, salient surface and surface variables such as plasticity and fatigue damage parameters.

\section{OTHER NUMERICAL MODELING METHODS}

Besides using the FEA other numerical modeling method are applied to predict fretting wear process.

Reference [15] described a numerical method based on quadratic programming to analyze fretting contacts, which incorporation of wear into a fretting analysis. Results obtained have a good agreement with FEA simulations undertaken by reference [1] and analytical fully worn solution.

Reference [16] presented a 3-D BEM formulation with Archard wear model for simulating 3-D fretting wear problems, without remeshing the surface of solids. The comparisons between BEM method and FE method and analytical models presented in literature have a good agreement.

Reference [17] presented a combined FDEM to modeling fretting wear based on Archerd wear model. The FEA is used to calculate the internal stresses of the contacting bodies, and discrete element method is employed to determine the interaction between the bodies. By comparing to pressure and frictional shear stress of continuum mechanics solution, the FDEM is validated.

\section{CONCLUSIONS}

Archard wear model and dissipated energy method are the most popular wear model to predict the process of fretting wear in literature. However, the Archard analysis is suitable to friction pairs displaying a stable friction evolution. Based on literature, it is shown that the dissipated energy method is more stable and permits a finer interpretation of the wear processes, whatever using FEA [13] or FDEM [18].

FDEM has a significant advantage over the FEA for modeling the debris, which play an important role at process of fretting wear.

\section{ACKNOWLEDGEMENTS}

The authors would like to acknowledge the support of China Scholarship Council.

\section{REFERENCES}

[1] McColl, I. R., Ding, J., Leen, S. B, Finite Element Simulation and Experimental Validation of Fretting Wear, Wear, 256 (11-12), 1114-1127, 2004.

[2] Blanchet, T.A., The Interaction of Wear and Dynamics of a Simple Mechanism, ASME Journal of Tribology, 119 (3), 597-599, 1997. 
[3] Hegadekatte, V., Huber, N., Kraft, O., Finite Element Based Simulation of Dry Sliding Wear, Modelling and Simulation In Materials Science and Engineering,13 (1), 57-75, 2005.

[4] Ding, J., Leen, S. B., McColl, I. R., The Effect of Slip Regime on Fretting Wear-Induced Stress Evolution, International Journal of Fatigue, 26 (5), 521-531, 2004.

[5] Ding, J., McColl, I. R., Leen, S. B., Shipway, P. H., a Finite Element Based Approach to Simulating the Effects of Debris on Fretting Wear, Wear, 263 (1-6), 481-491, 2007.

[6] Paulin, C., Fouvry, S., Meunier, C., Finite Element Modelling of Fretting Wear Surface Evolution Application to A Ti-6A1-4V Contact, Wear, 264 (1-2), 26-36, 2008.

[7] Stéphanie, B., Eva, H., Georges, C., Numerical Simulation of the Third Body in Fretting Problems, Wear, 270 (11-12), 876-887, 2011.

[8] Archard, J.F., Contact and Rubbing of Flat Surface. Jounal of Applied physics. 24 (8), 981-988, 1953.

[9] Madge, J. J., Leen, S. B., Shipway P. H., the Critical Role of Fretting Wear in the Analysis of Fretting Fatigue, Wear, 263 (1-6), 542-551, 2007.

[10] Mohd Tobi, A. L., Ding, J., Bandak, G., Leen, S. B., Shipway P. H., A Study on the Interaction Between Fretting Wear and Cyclic Plasticity for Ti-6Al-4V, Wear, 267 (1-4), 270-282, 2009.

[11] Fouvry, S., Paulin, C., Liskievicz, T., Application of an Energy Wear Approach to Quantity Fretting Contact Durability: Introduction of a Wear Energy Capacity Concept, Tribology International, 40 (10-12), 1428-1440, 2007.

[12] Paulin, C., Fouvry, S., Deyber, S., Wear Kinetics of Ti-6Al-4V Under Constant and Variable Fretting Sliding Conditions, Wear, 259 (1-6), 292-299, 2005.

[13] Paulin, C., Fouvry, S., Meunier, C., Finite Element Modelling of Fretting Wear Surface Evolution: Application to A Ti-6Al-4V Contact, Wear, 264 (1-2), 26-36, 2008.

[14] Zhang, T., McHugh, P.E., Leen, S.B., Computational Study on the Effect of Contact Geometry on Fretting Behaviour, Wear, 271 (9-10), 1462-1480, 2011.

[15] Nowell, D., Simulation of Fretting Wear in Half-Plane Geometries-Part II: Analysis of the Transient Wear Problem Using Quadratic Programming, Journal of Tribology, 132 (2), 021402-1-021402-8, 2010.

[16] Rodriguze-Tembleque L., Abascal, R., Aliabadi, M.H., a Boundary Elements Formulation for 3D Fretting-Wear Problems, Engineering Analysis with Boundary Elements, 35 (7), 935-943, 2011.

[17] Leonard, B. D., Patil, P., Slack, T.S., Sadeghi F., Shinde S., Mittelbach M., Fretting Wear Modeling of Coated and Uncoated Surfaces Using the Combined Finite Discrete Elemeng Method, Journal of Tribology, 133 (2), 021601-1-021601-12, 2011.

[18] Leonard, B. D., Sadeghi, F., Shinde, S., Mittelbach M., A Numerical and Experimental Investigation of Fretting Wear and a New Procedure for Fretting Wear Maps, Tribology Transactions, 55 (3), 313-324, 2012. 